\title{
Singlet-triplet filtering and entanglement in a quantum dot structure
}

\author{
G. Giavaras, ${ }^{1,2}$ J. H. Jefferson, ${ }^{2}$ M. Fearn, ${ }^{2}$ and C. J. Lambert ${ }^{1}$ \\ ${ }^{1}$ Department of Physics, Lancaster University, Lancaster LA14YB, England \\ ${ }^{2}$ QinetiQ, St.Andrews Road, Malvern WR143PS, England
}

(Dated: April 19, 2018)

\begin{abstract}
We consider two interacting electrons in a semiconductor quantum dot structure which consists of a small dot within a larger dot, and demonstrate a singlet-triplet filtering mechanism which involves spin-dependent resonances and can generate entanglement. By studying the exact time evolution of singlet and triplet states we show how the degree of both filtering and spin entanglement can be tuned using a time-dependent gate voltage.
\end{abstract}

PACS numbers:

In semiconductor quantum dots which can be formed for example at the interface of a gated GaAs/AlGaAs heterostructure, we can trap electrons and control their number precisely down to one $1,2,3,4$. The fact that the electrons in the dots are well-confined within a region of some nanometers and they are therefore isolated from the remaining environment of the host material, offers not only the opportunity to explore atomic-like effects and electron correlations $s^{2,3,5,6}$ in the solid state, but also to consider the quantum dots as promising candidates for nanoelectronic applications. For instance the proposal for realisation of spin and charge qubits in double quantum $\operatorname{dots}^{7}$ has attracted a lot of interest both theoretically ${ }^{8,9,10,11,12}$ and experimentally ${ }^{13,14,15,16}$ and the demonstration of entanglement, which is a necessary ingredient for two-qubit gates, is one of the main goals. Of course many other systems which include quantum dots make use of the Loss and DiVincenzo ${ }^{7}$ exchange-energy mechanism based on the Coulomb interaction to generate spinentanglement, such as for example flying qubits in surface acoustic wave structures ${ }^{17}$ and static-flying qubits in quantum wires ${ }^{18,19}$. The singlet-triplet qubit in a double quantum dot is also a promising candidate for quantum computation. The two-electron singlet-triplet $\left(S_{z}=0\right)$ states constitute the twolevel quantum system and as has been shown this scheme, which is also under experimental investigation ${ }^{16}$, is efficient for universal quantum gates ${ }^{20,21}$.

In this paper we propose and investigate a semiconductor dot structure which can induce a mechanism to filter singlettriplet states and thus to generate maximal spin entanglement between two electrons. The operation is controlled by a timedependent gate voltage which can tune the degree of filtering and entanglement.

To be specific, we choose material parameters for GaAs and consider the quasi-one-dimensional quantum dot structure which is shown in Fig. 1) (a), loaded with two electrons. It consists of a small open dot which is formed in the centre of a much larger dot. The small dot is such that it can bind only one electron and this means that the Coulomb interaction forces the second electron to occupy the region of the large dot. The latter has width $L=800 \mathrm{~nm} \sim 80 a_{B}^{*}$, where $a_{B}^{*}$ is the effective Bohr radius for GaAs. In this regime the two electrons are in the strong correlation regime ${ }^{22,23}$ i.e. the Coulomb interaction dominates over the kinetic energy and in the ground state the two electrons are localised in regions for which the electrostatic repulsion is minimised. Note that this is the case for both singlet and triplet states which give virtually the same electron distribution and they are separated with a very small antiferromagnetic exchange energy $J$.

To explicitly demonstrate and analyse these effects we have studied the two-electron problem with exact diagonalisation ${ }^{24}$ considering the parabolic-band Hamiltonian

$$
H=\sum_{i=1,2}\left[-\frac{\hbar^{2}}{2 m^{*}} \frac{\partial^{2}}{\partial x_{i}^{2}}+V_{d}\left(x_{i}\right)\right]+V_{c}\left(x_{1}, x_{2}\right),
$$

where $m^{*}=0.067 m_{o}$ is the effective mass of the electrons for GaAs. We have modelled the quantum dot with the Gaussian confining potential $V_{d}(x)=-V_{o} \exp \left(-x^{2} / 2 l_{o}^{2}\right)$, for $400 \mathrm{~nm} \leq \mathrm{x} \leq 400 \mathrm{~nm}$ and $V_{d}(x)=\infty$ otherwise $^{25}$. The parameters $V_{o}, l_{o}$ determine the depth and width of the small dot respectively and they are chosen to give only a single bound energy level $\sim-1.5 \mathrm{meV}$. The Coulomb term is given by $V_{c}\left(x_{1}, x_{2}\right)=q^{2} / 4 \pi \epsilon_{r} \epsilon_{o} r$, with $r=\sqrt{\left(x_{1}-x_{2}\right)^{2}+\lambda_{c}^{2}}$ and $\epsilon_{r}=13$ the relative permittivity in GaAs. This simplified form of the Coulomb interaction assumes that all excitations take place in the $x$-direction, whereas in the $y, z$-directions the electrons occupy at all times the lowest transverse modes. To satisfy this assumption we choose for all the calculations $\lambda_{c}=20 \mathrm{~nm}$, which gives a physical confinement length in the transverse $y, z$ directions much smaller than that in $x$. The two-electron time-independent Schrödinger equation is solved numerically by the configuration interaction method. The low-energy eigenstates consist of two closely spaced singlets and two triplets with a somewhat larger energy gap to higherlying states. Fig. 1(b) shows the lowest triplet eigenstate $\Phi_{o}^{T}\left(x_{1}, x_{2}\right)$ (antisymmetric) and Fig. 11 c) typical energy levels. The corresponding effective charge density of $\Phi_{o}^{T}\left(x_{1}, x_{2}\right)$ which is derived by integrating the two-electron charge density over the spatial coordinates of one of the two electrons i.e. $\rho^{T}(x)=2 q \int\left|\Phi_{o}^{T}\left(x, x^{\prime}\right)\right|^{2} d x^{\prime}$ is shown in Fig. 1 a). For this distribution one electron is bound in the small dot, whereas the second electron is at the left and right corners with equal probability due to the symmetric potential. The charge densities associated with all states in the low-lying manifold are in fact virtually identical to that shown in Fig. 1 (a).

This can be understood from a simple Heitler-London picture using one electron states derived from a Hartree approximation in which we replace the trapped electron in the small dot with an effective Hartree potential $V_{H}(x)=$ 

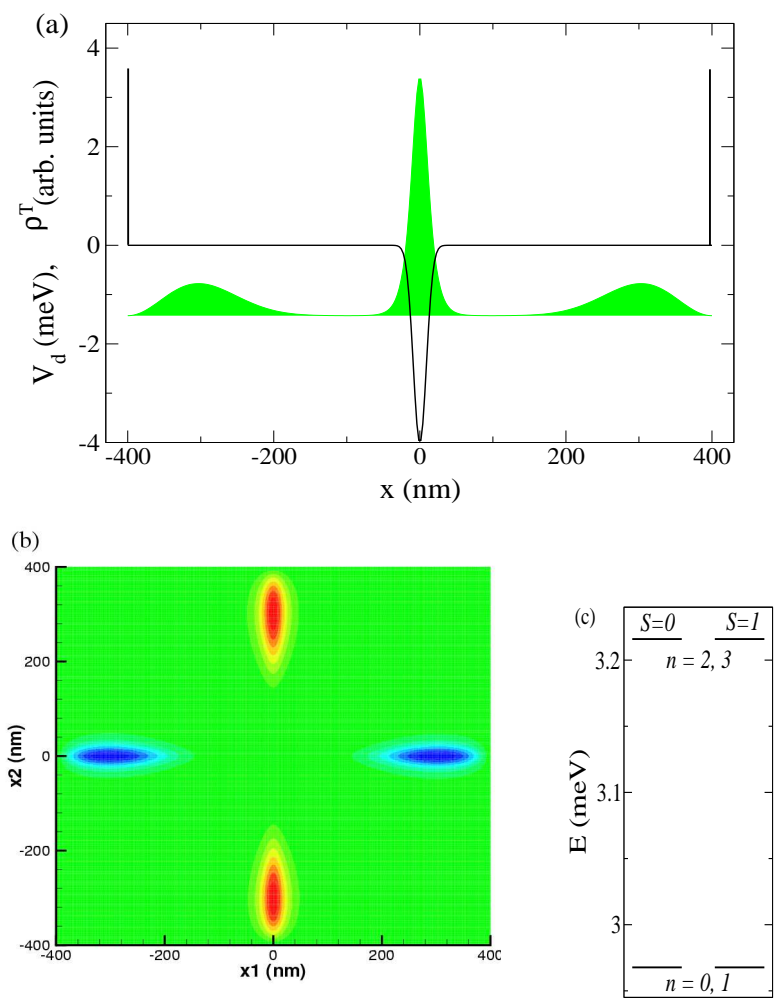

FIG. 1: (Color online) (a) Quantum dot confining potential and effective charge density (in arbitrary units) of the lowest triplet eigenstate. (b) Contour plot of the lowest triplet eigenstate. (c) The two-lowest singlet and triplet pairs.

$\int V_{c}\left(x, x^{\prime}\right)\left|\psi_{c}\left(x^{\prime}\right)\right|^{2} d x^{\prime}$, where $\psi_{c}(x)$ describes the single electron state of the small dot. The second electron feels the approximate potential $V_{H}(x)+V_{d}(x)$, which for the lowest two-electron states of interest acts as a double-well potential. The two lowest single-electron states $\psi_{-}(x), \psi_{+}(x)$, (bonding-antibonding) of this potential have a small energy splitting and peak in the left and right corners. Using the one electron Hartree states we may form the two lowest electron states (independent of spin) $\Phi_{o}\left(x_{1}, x_{2}\right)=\psi_{-}\left(x_{1}\right) \psi_{c}\left(x_{2}\right)$ and $\Phi_{1}\left(x_{1}, x_{2}\right)=\psi_{+}\left(x_{1}\right) \psi_{c}\left(x_{2}\right)$ and similarly for the next higher pair. Within the Hartree model this means that not only the lowest singlet, triplet eigenstates have a distribution of the form of Fig. 1 (a), but also the first excited eigenstates which indeed agrees with the results that we derive from the exact diagonalisation. Note that even though the Hartree model gives great insight into the two-electron energies and distributions of the low-lying states, with remarkably little effort, the symmetric and antisymmetric combinations $\Phi^{S, A}\left(x_{1}, x_{2}\right)=\left[\Phi_{o}\left(x_{1}, x_{2}\right) \pm \Phi_{o}\left(x_{2}, x_{1}\right)\right] / \sqrt{2}$ give a poor approximation to the singlet-triplet splitting, with even the incorrect sign. This is little improved by using more accurate one-electron states, e.g. from Hartree-Fock or DFT, being a consequence of strong correlations requiring accurate solutions of the two-electron problem. However, as we discuss later, the system may be described accurately by an extended Hubbard model.

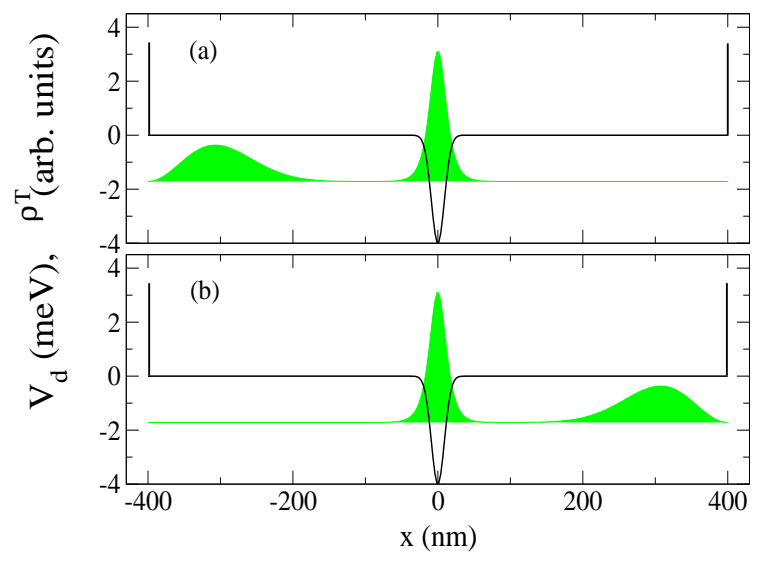

FIG. 2: (Color online) Effective charge density for (a) left and (b) right triplet states. The quantum dot confining potential is also shown.

For the dynamics that we describe below it is important to introduce left and right states which are formed by combining the two lowest eigenstates for singlet $\Phi_{L}^{S}=\Phi_{o}^{S}+\Phi_{1}^{S}$, $\Phi_{R}^{S}=\Phi_{o}^{S}-\Phi_{1}^{S}$ and triplet $\Phi_{L}^{T}=-\Phi_{o}^{T}+\Phi_{1}^{T}, \Phi_{R}^{T}=\Phi_{o}^{T}+\Phi_{1}^{T}$ (unnormalised). For these states one electron is bound in the small dot whereas the second electron is localised to the corresponding corner as we demonstrate in Fig. 2 2 for the triplet.

Although the effective charge density of the low-lying singlets and triplets is virtually identical, the wave functions are quite different and give rise to effective interactions between spins, responsible for their entanglement. To demonstrate how these states can generate spin-entanglement and give rise to singlet-triplet filtering, we first prepare the system such that one electron is located on the left with spin up and the other in the central dot with spin down. A suitable non-entangled state of this type may be written as a superposition of the left singlet and the left $S_{z}=0$ triplet state i.e. $\Psi_{\uparrow \downarrow}=\left(\Phi_{L}^{S} \chi_{\uparrow \downarrow}^{S}+\Phi_{L}^{T} \chi_{\uparrow \downarrow}^{T}\right) / \sqrt{ } 2$, with the spin eigenstates $\chi_{\uparrow \downarrow}^{S / T}(1,2)=\left[\chi_{\uparrow}(1) \chi_{\downarrow}(2) \mp \chi_{\downarrow}(1) \chi_{\uparrow}(2)\right] / \sqrt{ } 2$. An approximation to this state may be obtained in principle by applying a small source-drain bias, $V_{s d}$, across the quantum dot structure and allowing the system to relax to its ground state (singlet). The spins may then be initialised using magnetic fields and a microwave pulse, as suggested for scalable qubit arrays ${ }^{7,13}$. If $V_{s d}$ is removed, then oscillations (in a similar fashion to a double dot system) and entanglement will develop with a typical time for the triplet component of the wave function on the order of $\pi \hbar / \Delta E^{T} \sim 1 \mu \mathrm{s}$, where $\Delta E^{T}=E_{1}^{T}-E_{o}^{T}$ is the energy splitting of the two lowest triplet eigenstates when $V_{s d}=0$ (similarly for the singlet). To speed-up the process we can apply a time-dependent gate voltage that will tune in such a way the dot potential so that to increase this energy splitting. In this work we have modelled the gate voltage potential using the expression $V_{g}(x, t)=-V_{p}(t) \exp \left(-x^{2} / 2 l_{g}^{2}\right),\left(l_{g} \sim 130\right.$ 
$\mathrm{nm})$ which is driven by a triangular pulse of the form

$$
V_{p}(t)=\left\{\begin{array}{cc}
\frac{2 V_{b}}{T_{p}} t, & 0 \leq t \leq T_{p} / 2 \\
-\frac{2 V_{b}}{T_{p}} t+2 V_{b}, & T_{p} / 2 \leq t \leq T_{p},
\end{array}\right.
$$

where $T_{p}$ is the period of the pulse and $V_{b}$ the maximum gate voltage. We have studied the case for which the gating rate $\alpha=\left|d V_{p} / d t\right|$ is such that to a good approximation only the two lowest singlet and two lowest triplet states are involved in the dynamics. During the first half of the cycle $\left(0 \leq t \leq T_{p} / 2\right)$ the effect of the voltage is to decrease the effective width of the dot thereby increasing the energy splitting and the interaction, whereas during the second half of the cycle $\left(T_{p} / 2 \leq \mathrm{t}\right.$ $\leq T_{p}$ ) the process is reversed.

The dynamics of the two electrons is governed by the timedependent Schrödinger equation with the Hamiltonian (1) and for a total potential $V_{d}(x)+V_{g}(x, t)$ which is the sum of the dot confining potential and the time-dependent gate potential. For $t>0$, the spin eigenstates are unchanged for singlet or triplet components, whereas the evolution of the corresponding orbital states is given directly by the solution of the time-dependent Schrödinger equation which is implemented numerically using the staggered-time algorithm proposed by Visscher ${ }^{26}$. The time evolution of the initial state, $\Psi_{\uparrow \downarrow}$, is then determined by adding the separately determined singlet and triplet components. In Fig. 3 we show two examples of the final electron distribution for singlet and triplet components. Specifically, in Fig. 3 a) the singlet corresponds to a good approximation to the right state, whereas the triplet to the left state. This is the ideal filtering regime (together with the opposite limit) in which at the final time the two components occupy different spatial regions. Note that in this regime the two electrons are fully entangled provided the measurement domain is restricted either to the left or right region, detecting the singlet or the $S_{z}=0$ triplet state respectively, which are fully entangled states. Performing an additional measurement but initialising the electron spins to a purely triplet state (either of $S_{z}= \pm 1$ ) reveals the occupation region of the triplet state hence offering a way to discriminate between singlet and triplet components. Note that in the most general case which is shown in Fig. 3 b) the final two-electron state is a superposition of left and right states for both singlet and triplet components. Clearly, a measurement with restriction to either the left domain or the right domain would probe a partially entangled state.

To understand the dynamics and the difference between singlet and triplet components we can write the time-dependence, for example of the singlet, using the corresponding two lowest energy states at all times i.e. $\Phi^{S}=C_{L}^{S} \Phi_{L}^{S}+C_{R}^{S} \Phi_{R}^{S}$ in the left-right basis with the amplitudes $C_{L}^{S}=e^{-i \varphi^{S}} \cos \omega^{S}$ and $C_{R}^{S}=i e^{-i \varphi^{S}} \sin \omega^{S}$. The parameters $\varphi^{S}=\int_{0}^{t}\left[E_{1}^{S}\left(t^{\prime}\right)+\right.$ $\left.E_{o}^{S}\left(t^{\prime}\right)\right] d t^{\prime} / 2 \hbar$ and $\omega^{S}=\int_{0}^{t}\left[E_{1}^{S}\left(t^{\prime}\right)-E_{o}^{S}\left(t^{\prime}\right)\right] d t^{\prime} / 2 \hbar$ depend on the two lowest energy levels and change with time. Note that $\omega^{S}$ determines the amount of filtering because it determines the period of the oscillations between left and right states, whereas both $\varphi^{S}$ and $\omega^{S}$ determine the degree of entanglement as we demonstrate below. In Fig. 4 we show the

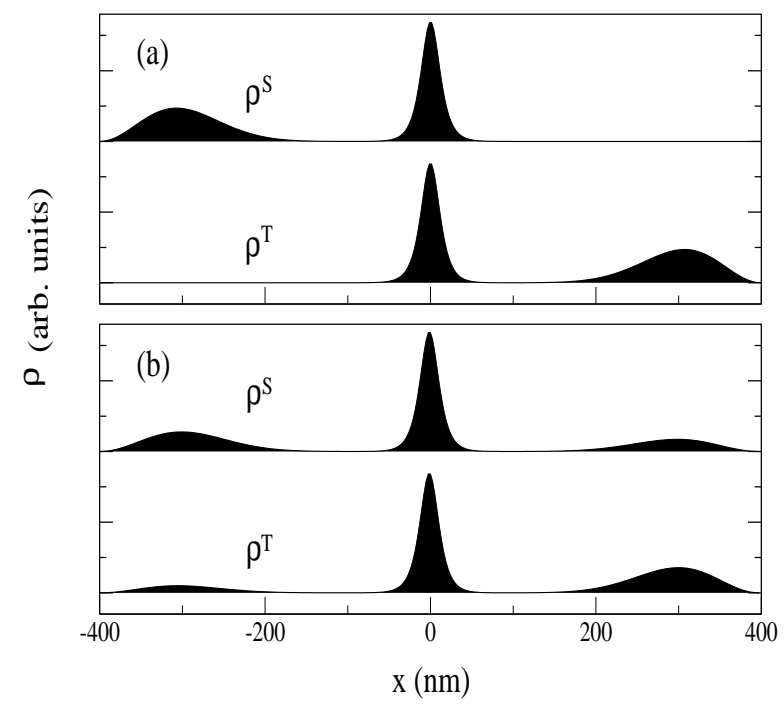

FIG. 3: Effective charge density for singlet and triplet components at the final time for (a) a case in the filtering regime and (b) the most general case.

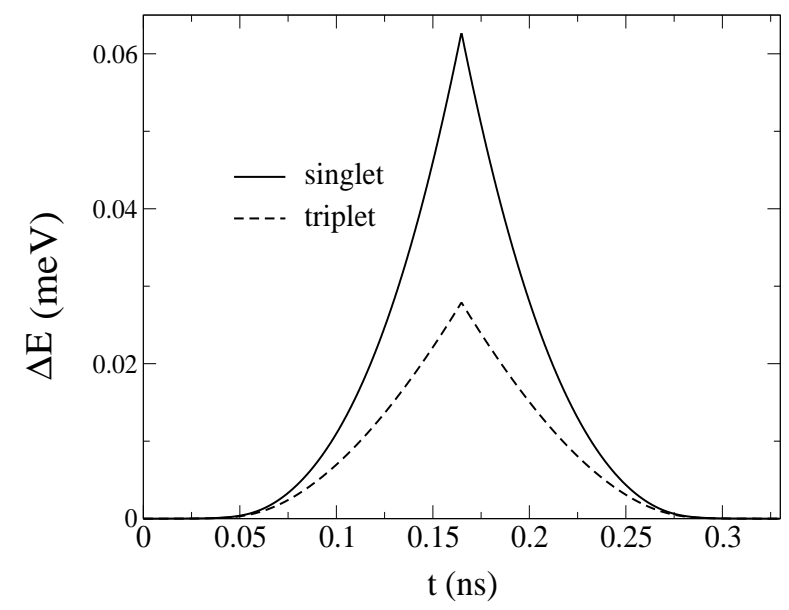

FIG. 4: Energy splitting of the two-lowest levels for singlet and triplet components, as a function of time for one cycle in the filtering regime.

energy splitting of the two lowest levels (the energies are calculated from instantaneous solutions) as a function of time for one cycle in the filtering regime which gives fully entangled electrons in time $T_{p} \sim 0.3 \mathrm{~ns}$ (for $V_{b} \sim 2.8 \mathrm{meV}$ ). We see that the splitting is larger for the singlet and this means that $\omega^{S}>\omega^{T}$ i.e. the singlet component oscillates faster than the triplet. We can understand this effect with a Hartree approximation: because the small dot has only one bound energy level, $\varepsilon_{c}$, the tunneling time (the time that the electron in the left corner needs in order to move to the right corner) for the triplet is longer due to the Pauli blocking. Within the Hartree model the electron in the large dot needs to tunnel through an effective double barrier due to the Coulomb repulsion from 


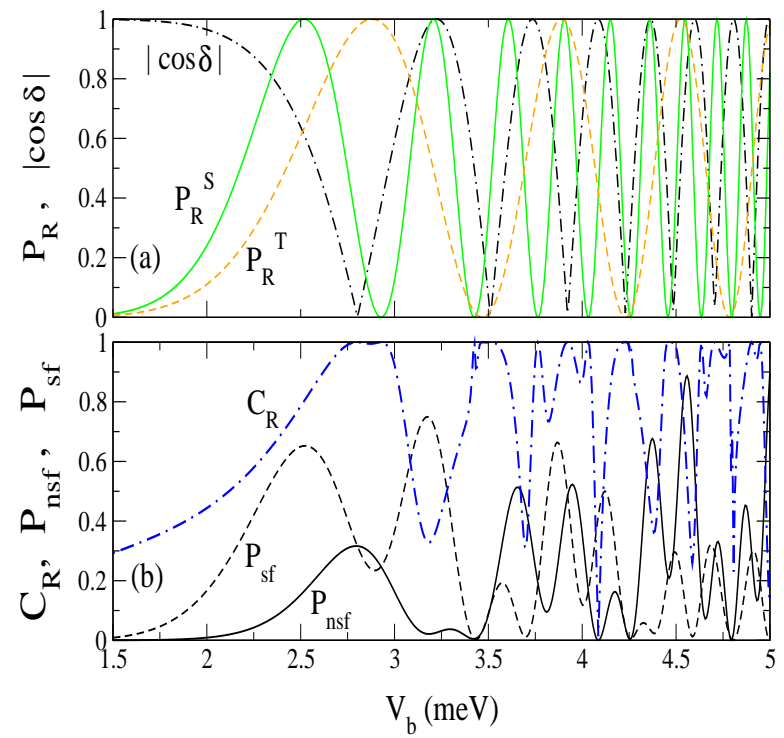

FIG. 5: (Color online) Dependence (at the final time), as a function of maximum gate voltage for fixed gating rate, of: (a) absolute value of $\cos \delta$ and right probabilities for singlet and triplet components; (b) concurrence, spin-flip and non spin-flip probabilities calculated for the right region.

the trapped electron in the small dot, which has only one resonance energy level $\sim \varepsilon_{c}+U_{c}$ (with $U_{c}$ the Coulomb energy when both electrons occupy $\varepsilon_{c}$ ) and corresponds to a singlet state. For a triplet resonance to exist the small dot needs to have at least two bound levels due to the Pauli principle. It is worth noting that the efficiency of this singlet-triplet filtering mechanism and the induced entanglement has been also demonstrated in scattering problems between a static and a flying qubit ${ }^{18,19}$.

To quantify the degree of entanglement which arises as a consequence of the interaction when the electrons are close together we have calculated concurrence ${ }^{27}$ at the final time, from spin-flip, $P_{s f}$, and non spin-flip, $P_{n s f}$, probabilities. For example at the right region the amplitudes for these probabilities are

$$
C_{n s f}=\frac{C_{R}^{T}+C_{R}^{S}}{2}, \quad C_{s f}=\frac{C_{R}^{T}-C_{R}^{S}}{2},
$$

which are derived by writing the corresponding final state $\Psi_{\uparrow \downarrow}\left(t_{f}\right)$ in the basis of spin eigenstates. Concurrence at the right region is then given by

$$
C_{R}=\frac{2\left(P_{n f f} P_{s f}\right)^{1 / 2}}{P_{s f}+P_{n s f}}
$$

with $P_{n s f}=\left|C_{n s f}\right|^{2}$ and $P_{s f}=\left|C_{s f}\right|^{2}$, thus

$$
C_{R}=\frac{\left[\left(P_{R}^{S}+P_{R}^{T}\right)^{2}-4 P_{R}^{S} P_{R}^{T} \cos ^{2} \delta\right]^{1 / 2}}{P_{R}^{S}+P_{R}^{T}},
$$

where we have set $P_{R}^{S}=\left|C_{R}^{S}\right|^{2}=\sin ^{2} \omega^{S}$ and $P_{R}^{T}=$ $\left|C_{R}^{T}\right|^{2}=\sin ^{2} \omega^{T}$ and the relative phase $\delta=\varphi^{S}-\varphi^{T}$. A (a)
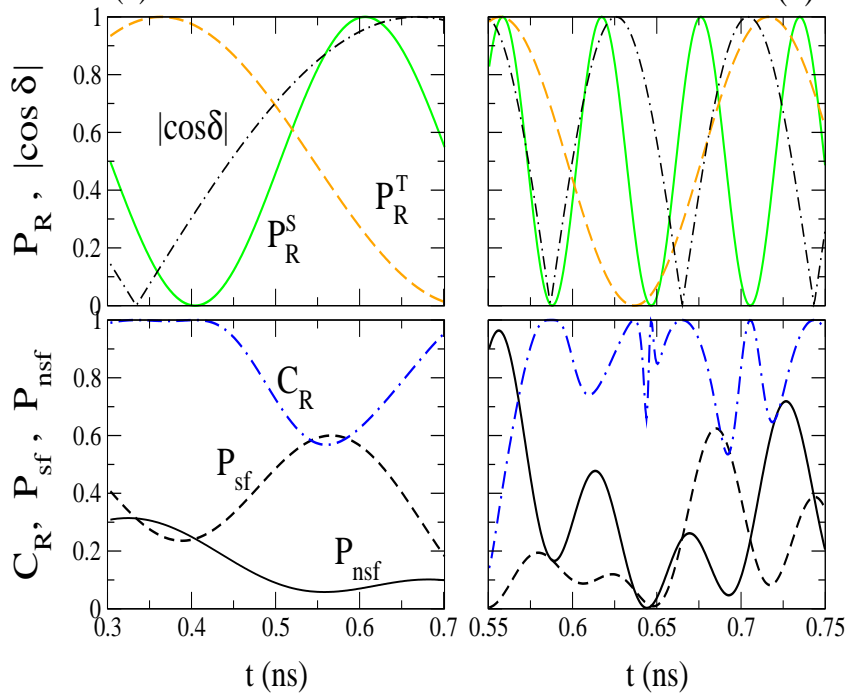

FIG. 6: (Color online) Dependence (at the final time) of quantities in Fig.5 as a function of pulse duration time for two different maximum gate voltages. (a) $V_{b}=2.75 \mathrm{meV}$ and (b) $V_{b}=5 \mathrm{meV}$.

similar expression can be derived for the left and even for the total region. Note that by definition $0 \leq C_{R} \leq 1$ where the limit $C_{R}=0$ corresponds to unentangled electrons, whereas the limit $C_{R}=1$ to fully entangled electrons. The measurement in the right region is meaningful only when $P_{R}^{S} \neq 0$, and/or $P_{R}^{T} \neq 0$ i.e. when the right state is occupied for singlet and/or triplet. We see from Eq. (4) that $C_{R}=1$ when $P_{s f}=P_{n s f}$ or equivalently from Eq. (5) when $\cos \delta=0$ and/or when $P_{R}^{S}=0$ and simultaneously $P_{R}^{T} \neq 0$ or vice versa.

In Fig. 5 we present the dependence of probabilities, phase shift and concurrence as a function of maximum gate voltage $V_{b}$ for a fixed gating rate $\alpha \sim 18 \mathrm{meV} / \mathrm{ns}$ which ensures that to a good approximation only the two lowest eigenstates for both singlet and triplet components are involved into the dynamics. Figure 5(a) shows the probabilities $P_{R}^{S}, P_{R}^{T}$ and the absolute magnitude of the quantity $\cos \delta$ calculated at the final time, versus $V_{b}$. We see that the probabilities $P_{R}^{S}, P_{R}^{T}$ do not oscillate for small gates voltages $V_{b} \lesssim 2.5 \mathrm{meV}$ (but rather they increase) because the energy splitting of the two-lowest levels in this limit remains relatively small and therefore the tunneling time long. Further increase of the gate voltage induces welldefined oscillations. In particular the probability of the singlet component oscillates faster than the triplet because the energy splitting which determines the frequency of the oscillations is larger for the singlet. Note also that the frequency of oscillations increases with gate voltage for both components following the increase of the energy splitting. Figure 5(b) shows the concurrence $C_{R}$ and the spin-flip $P_{s f}$ and non spin-flip $P_{n s f}$ probabilities calculated at the final time. When these probabilities are equal the entanglement is maximum whereas when one of them is zero the electrons remain unentangled. For a gate voltage $V_{b} \sim 2.8 \mathrm{meV}$ we have an example of the ideal filtering regime for which $P_{R}^{S} \sim 0, P_{R}^{T} \sim 1$ and $P_{s f}=P_{n s f}$ giv- 


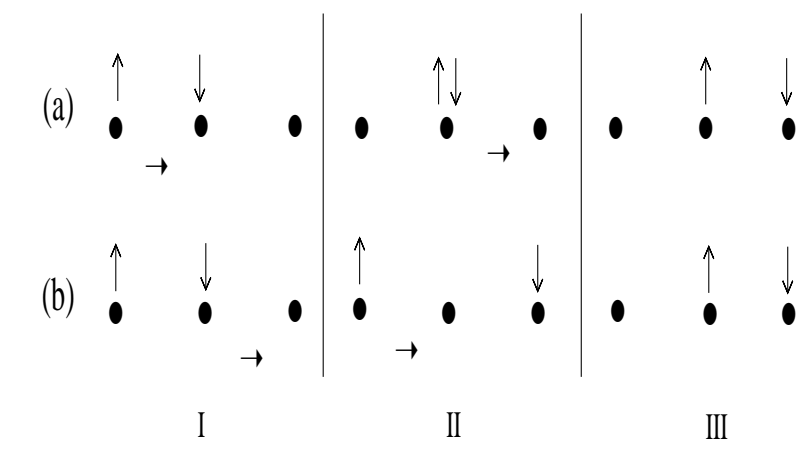

FIG. 7: Illustration of the two-electron spin dynamics in a three-step process I, II, III. The process (a) is allowed only for singlets whereas the process (b) is allowed for both singlets and triplets.

ing $C_{R}=1$, since only a triplet state occupies the right region. The generic condition for ideal filtering is when $\sin \omega^{S}=1$ and simultaneously $\sin \omega^{T}=0$ or vice versa. Other filtering cases (non-ideal) which give $C_{R}=1$ occur when one of the components is zero, say $P_{R}^{S}=0$ and the other is non-zero $P_{R}^{T} \neq 0$, but not however equal to one. Finally an interesting limit is when $P_{R}^{S}=P_{R}^{T}$ for which the concurrence reduces to the simple expression $C_{R}=|\sin \delta|$ which depends only on the relative phase between singlet and triplet components.

Figure 6 presents the dependence of various quantities as a function of the pulse duration time for two different gate voltages. As we see by comparing Figs. 6(a) and 6(b) the period of the oscillations can be controlled with the value of the maximum gate voltage with the larger $V_{b}$ inducing faster oscillations due to the larger energy splitting.

Finally, we point out that the low-lying spin states relevant to the dynamics of this entangler which involve virtually identical charge distributions at the left, centre and right of the dot structure suggest that this charge-spin system may be modelled by a three-site Hubbard model of the form

$$
\begin{aligned}
H_{e f f}= & \varepsilon\left(n_{L}+n_{R}\right)+\varepsilon_{c} n_{C} \\
& +\gamma \sum_{\sigma, i=L, R}\left(C_{i, \sigma}^{\dagger} C_{C, \sigma}+\text { h.c. }\right) \\
& +U\left(n_{L, \uparrow} n_{L, \downarrow}+n_{R, \uparrow} n_{R, \downarrow}\right)+U_{c} n_{C, \uparrow} n_{C, \downarrow} \\
& +V\left(n_{L} n_{C}+n_{C} n_{R}\right),
\end{aligned}
$$

where $n_{i}=\sum_{\sigma} n_{i, \sigma}=\sum_{\sigma} C_{i, \sigma}^{\dagger} C_{i, \sigma}, i=L, R, C$ and
$C_{i, \sigma}^{\dagger}\left(C_{i, \sigma}\right)$ creates (destroys) an electron on site $i$ with spin$\sigma$. The on-site orbital energy for the left and right sites is $\varepsilon$ and for the central site is $\varepsilon_{c}$. The on-site Coulomb energy is denoted by $U$ for the left and right sites and by $U_{c}$ for the central site. The nearest site Coulomb energy is $V$ and finally $\gamma$ expresses the hopping between nearest sites. Note that all the physical parameters $\left(\varepsilon, \varepsilon_{c}, U, U_{c}, V, \gamma\right)$ may be estimated from the Hartree approximation described earlier. Working within the restricted subspace for which the two-electron basis states consist of six singlets and three triplets, we can extract the correct energy splitting of the two-lowest eigenstates (for both singlet and triplet), the corresponding eigenstates and the antiferromagnetic exchange energy. Solution of this time-dependent model Eq. (6) does indeed show qualitatively the same behaviour as the original continuous problem but with considerable saving in computer time once the timedependent parameters are known. The analysis based on the effective Hamiltonian Eq. (6) indicates that the physical behaviour that we have demonstrated can also be realised in a triple quantum dot structure. More importantly, the Hubbard model gives insight into the behaviour of the system since it is readily mapped onto an effective charge-spin model for the low-lying manifold of two singlets and two triplets. In particular, for singlets there are two processes by which an electron may tunnel from left to right, as shown in Fig. 7 The process (a), that is only allowed for singlets, has amplitude $\Delta_{1}=\gamma^{2} /\left(U-V+\varepsilon_{c}-\varepsilon\right) \sim J$ where $J$ is the Heisenberg exchange energy between an electron spin on the central site and one on either the left or the right site. On the other hand, process (b) is valid for both singlets and triplets and has amplitude $\Delta_{2}=\gamma^{2} /\left(\varepsilon-\varepsilon_{c}-V\right)$. The relative rate of tunneling for singlet and triplet is thus tuned by the gate by changing $\varepsilon_{c}$, since making $\varepsilon_{c}$ more negative the energy denominator in $\Delta_{1}$ decreases whereas that of $\Delta_{2}$ increases.

In summary we have presented a quantum dot structure and described the two-electron distribution for the lowest singlet and triplet states. By studying the electron dynamics due to a time-dependent gate voltage we showed how we can induce a singlet-triplet filtering based on the Pauli blocking effect for the triplet state. This can generate full spin-entanglement within the order of $\sim 0.3 \mathrm{~ns}$. Both the degree of filtering and entanglement can be efficiently controlled with the gating rate and the maximum applied gate voltage.

G.G. thanks UK EPSRC for funding. This work was supported by the UK Ministry of Defence.
${ }^{1}$ L. P. Kouwenhoven, T. H. Oosterkamp, M. W. S. Danoeastro, M. Eto, D. G. Austing, T. Honda, and S. Tarucha, Science 278, 1788 (1997).

2 S. Tarucha, D. G. Austing, T. Honda, R. J. van der Hage, and L. P. Kouwenhoven, Phys. Rev. Lett. 77, 3613 (1996).

${ }^{3}$ L. P. Kouwenhoven, D. G. Austing, and S. Tarucha, Rep. Prog. Phys. 64, 701 (2001).

${ }^{4}$ W. G. van der Wiel, S. De Franceschi, J. M. Elzerman, T. Fujisawa, S. Tarucha, and L. P. Kouwenhoven, Rev. Mod. Phys. 75, 1
(2003).

5 D. H. Cobden and J. Nygard, Phys. Rev. Lett. 89, 046803 (2002).

${ }^{6}$ S. Moriyama, T. Fuse, M. Suzuki, Y. Aoyagi, and K. Ishibashi, Phys. Rev. Lett. 94, 186806 (2005).

7 D. Loss and D. P. DiVincenzo, Phys. Rev. A 57, 120 (1998).

${ }^{8}$ G. Burkard, D. Loss, and D. P. DiVincenzo, Phys. Rev. B 59, 2070 (1999).

9 J. Schliemann, D. Loss, and A. H. MacDonald, Phys. Rev. B 63, 085311 (2001). 
${ }^{10}$ X. Hu and S. Das. Sarma, Phys. Rev. B 61, 062301 (2000).

11 M. Friesen, P. Rugheimer, D. E. Savage, M. G. Lagally, D. W. van der Weide, R. Joynt, and M. A. Eriksson, Phys. Rev. B 67, 121301(R) (2003).

${ }^{12}$ P. Zhang, Q. K. Xue, X. G. Zhao, and X. C. Xie, Phys. Rev. A 66, 022117 (2002).

${ }^{13}$ F. H. L. Koppens, C. Buizert, K. J. Tielrooij, I. T. Vink, K. C. Nowack. T. Meunier, L. P. Kouwenhoven, and L. M. K. Vandersypen, Nature 442, 766 (2006).

${ }^{14}$ R. Hanson, L. H. Willems van Beveren, I. T. Vink, J. M. Elzerman, W. J. M. Naber, F. H. L. Koppens, L. P. Kouwenhoven, and L. M. K. Vandersypen, Phys. Rev. Lett. 94196802 (2005).

15 T. Hayashi, F. Fujisawa, H. D. Cheong, Y. H. Jeong, and Y. Hirayama, Phys. Rev. Lett. 91, 226804 (2003).

16 J. R. Petta, A. C. Johnson, J. M. Taylor, E. A. Laird, A. Yacoby, M. D. Lukin, C. M. Marcus, M. P. Hanson, A. C. Gossard, Science 309, 2180 (2005).

${ }^{17}$ C. H. W. Barnes, J. M. Shilton, and A. M. Robinson, Phys. Rev. B 62, 8410 (2000).

18 J. H. Jefferson, A. Ramšak, and T. Rejec, Europhys. Lett. 75, 764
(2006).

19 G. Giavaras, J. H. Jefferson, A. Ramšak, T. P. Spiller, and C. J. Lambert, Phys. Rev. B in press, cond-mat/0610168

${ }^{20}$ R. Hanson and G. Burkard, cond-mat/0605576 (2006).

${ }^{21}$ J. M. Taylor, H.-A.Engel, W. Dür, A. Yacoby, C. M. Marcus, P. Zoller, and M. D. Lukin, Nature Physics 1, 177 (2005).

${ }^{22}$ G. W. Bryant, Phys. Rev. Lett. 59, 1140 (1987).

23 C. E. Creffield, W. Häusler, J. H. Jefferson, and S. Sarkar, Phys. Rev. B 59, 10719 (1999).

${ }^{24}$ For two-electrons $\Psi\left(x_{1}, \sigma_{1}, x_{2}, \sigma_{2}\right)=\Phi\left(x_{1}, x_{2}\right) \chi\left(\sigma_{1}, \sigma_{2}\right)$ with $\Phi$ symmetric (antisymmetric) for singlet (triplet) under electron exchange. Because the Hamiltonian does not contain any spin dependent term we can consider only the spatial components.

25 We have used this potential form mainly for simplicity. Note however that the main results that we present do not depend qualitatively on this particular choice and they can be easily extended to more realistic potential profiles.

${ }^{26}$ P. B. Visscher, Comput. Phys. 5, 596 (1991).

27 W. K. Wootters, Phys. Rev. Lett. 80, 2245 (1998). 